\title{
Conception of Smart Home Perimeter Security System Based on Solar Powered IOT Solutions
}

\author{
Khudaybergenov Timur, YoungMin Kim, HyeJeong Cho, DeokGun Woo, Jaesang Cha
}

\begin{abstract}
This paper propose design a conception of Perimeter Security System we called "Outpost". General idea is organization of work of remote settled and independent powered security module using Raspberry Pi hardware platform and some IoT sensors. Software part is explained on the level of the diagrams and describing conception of Edge Computing on IoT microcontroller. Represented work describe of using of the suggested solution and providing information of powering of hardware. An experimental model was carried out with the purpose of Security IoT module designing. Description of working model which is able to connect to any existing Smart Home network is given in this paper
\end{abstract}

Keywords: IoT, Solar Energy, Smart Home, Raspberry Pi, IoT based Security Solution.

\section{INTRODUCTION}

A smart home is a complex of systems integrated into a single information space, ensuring maximum safety of people and property, maintaining comfortable working and resting conditions, and also allowing increasing the efficiency of services while reducing operating costs. The definition of "smart home", from our point of view, can only be applied to such automation solutions of modern buildings in which all systems are integrated into a single control complex. They are able to not only functionally solve their own problems, but also complement each other, function as parts of a single system.

Conventional security systems are keeping homes safe from inner and outer menace. A smart home security system, however, must offer much more benefits. Home automation technology notifies if any problems and moreover can analyze current situation and make a decision independently.[1]

Most of the security systems has a limitation as the indoor using only. Smart homes must be protected more than it. But for nowadays this systems are or still prototypes or extremely expensive.

\section{RELATED WORKS}

An intrusion detection systems as a smart security complex element is a wide theme for engineering researches all over the world. Many scientists and engineers are developing IoT

Revised Version Manuscript Received on 10 September, 2019.

Khudaybergenov Timur, Graduate School of Nano IT Design Fusion, Seoul National University of Science and Technology, Korea.

YoungMin Kim, Welfrun Co., Ltd, Korea

HyeJeong Cho, DepT. of Electronics and IT Media Engg., Seoul National University of Science and Technology, Korea.

DeokGun Woo, IoT Convergence Technology Institution., Seoul National University of Science and Technology, Korea.

Jaesang Cha,VTASK Co., Ltd, Korea.(email: vtask2018@gmail.com) based solutions for security systems. Md. Yousuf Hossain in the work IOT based Automated Intrusion Detection System, describing one of the possible methods of using IoT sensors to achieve this goal, through combining Paspberry $\mathrm{Pi}$ microcomputer and alerting system based on existing cellular network. Work describes an algorithms and principle conception of organization of communication between IoT sensors controller and existing cellular [2].

Some authors are describing the ways of how to solve the problems of intruders by using additional IoT sensors. For example Hyoung-Ro Lee in Design and Implementation of a Visitor Detection System using Hybrid Sensors, describing the using tandem of camera and ultrasonic sensor, when Deepak.S.Kumbhar in IoT Based Home Security System Using Raspberry Pi solving same problem by combining camera module and PIR based sensor

However the needs for the security are still high, and many solutions are under researches. We suggesting one of the possible solutions for this area.

\section{III. “OUTPOST" ALGORITHM}

Using of IoT based solution for a smart home security is challenge in an Edge Computing level. "Outpost" smart security system must have an ability to make a decision on reaction, to avoid fails and mistake actions. System should detect potential intruder by using IoT sensors set. When all the sensors state are fall under the trigger condition, it should make a decision to make a reaction on intruder, or/and send the data to the smart home main controller. "Outpost" system activity diagram is shown on Figure 1.

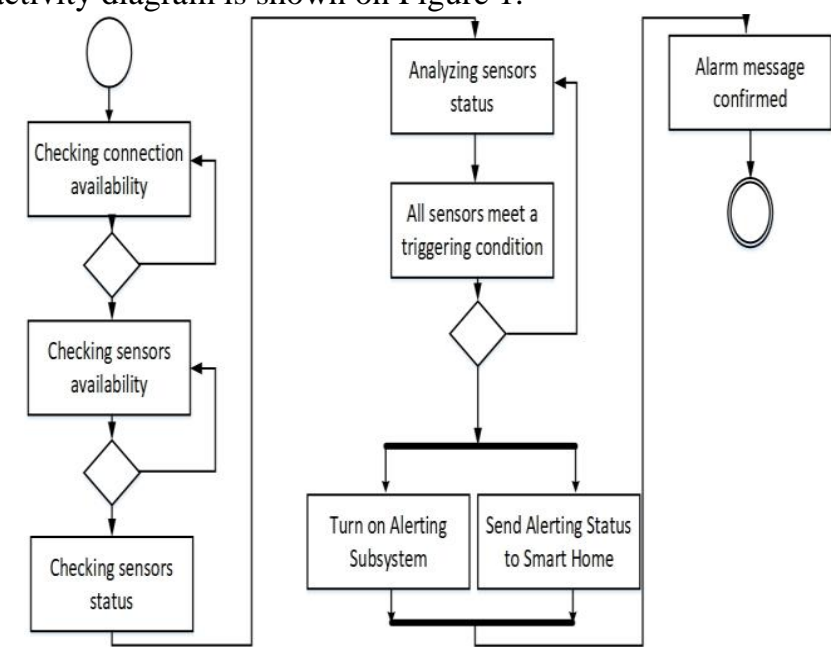

Figure 1. “Outpost” system activity diagram.

Published By:

Blue Eyes Intelligence Engineering

\& Sciences Publication 


\section{CONCEPTION OF SMART HOME PERIMETER SECURITY SYSTEM BASED ON SOLAR POWERED IOT SOLUTIONS}

\section{SOLAR POWER BASED IOT SOLUTION}

Nowadays more and more countries are changing street light infrastructure to be powered by solar energy. Currently, there is an increase in energy consumption, at the same time, there is an increase in electricity prices and the reserves of traditional resources are decreasing. In this regard, developments in the field of renewable energy are becoming relevant. Solar energy is one of the most actively developing types of renewable energy sources [3, 4]. The advantages using of solar panels are not only in an inexhaustible renewable energy source and no pollutants into the environment, but possibility to install them almost any convenient place and also solar panels allow you to provide energy to areas remote from the electrical grid [5]. Thant the main reason in this project we planning to use solar powered street light stands as the IoT "Outpost" for perimeter security of the smart house.

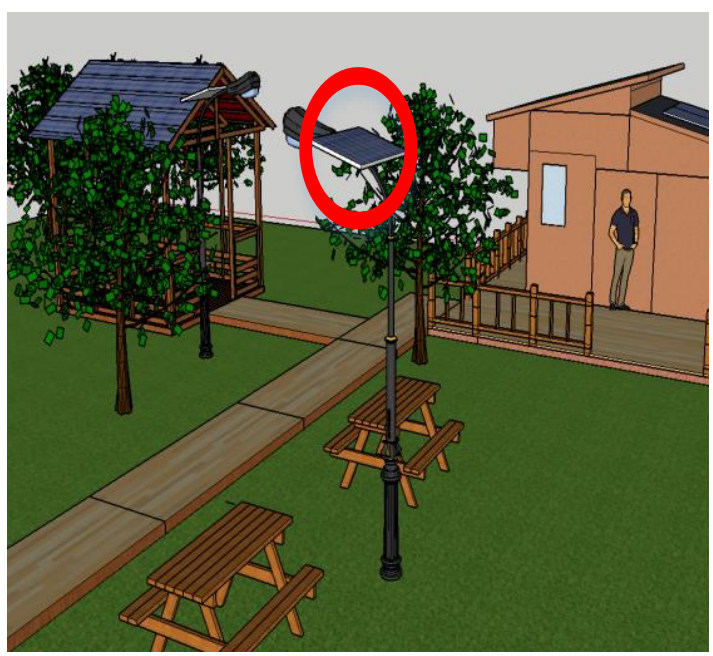

Figure 2. Solar panel powered Street light.

Solar panel provides enough power for supplying any IoT system, because mainly their power consumption is small. Suggested scheme could be directly connected to the power bank of the autonomous street light and need no SMPS modules. Instead of SMPS we planning to use a step down DC-DC convertors which are chip and provide enough current to power our system. Typical connection scheme for the solar powered street light will be as shown on the Figure 3.

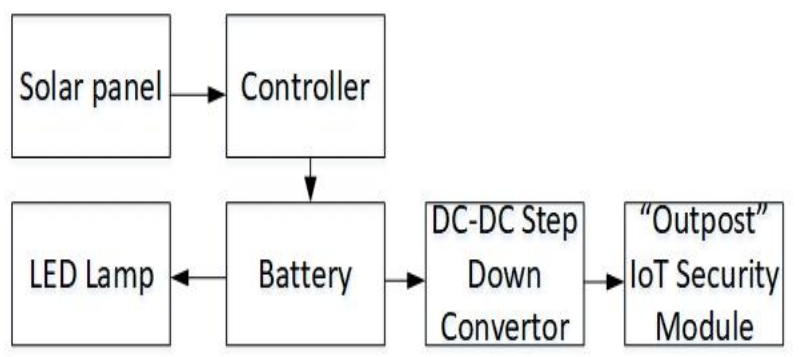

Figure 3. IoT Security module powering block diagram.

\section{DESIGN OF THE SYSTEM \& RESULTS}

In this work as the main controller of our IoT security module we have choose Raspberry Pi 3 microcomputer. Main reason of this is an opportunity to use this microcomputer as the edge computer element, enough numbers of GPIOs and flexibility provided by Python. As an experimental example of IoT security module we used human detection sensor based on PIR elements [9], camera, and ultrasonic sensor [8]. As pointed above "Outpost" system is using edge computing to identify potential intruder through analyzing data from list of sensors. This is made to prevent mistakes and fail-alarms. System hardware scheme is shown on a Figure 4.

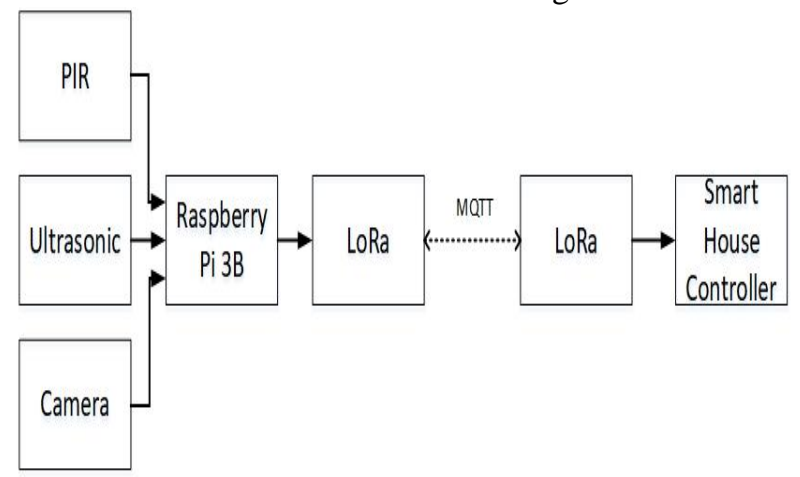

Figure 4. Hardware scheme of proposed system

System interconnection between smart home controller and remote IoT Security module in current project is realized on LoRa communication, to achieve the lowest power consumption on signal transmitting [8, 9]. In real conditions connection type could easily be changed to any available communication method. As communication protocol between the systems here suggested a MQTT protocol, due easy convergence and extending. State diagram of Smart home controller and "Outpost" solution intercommunication is shown on Figure 5.

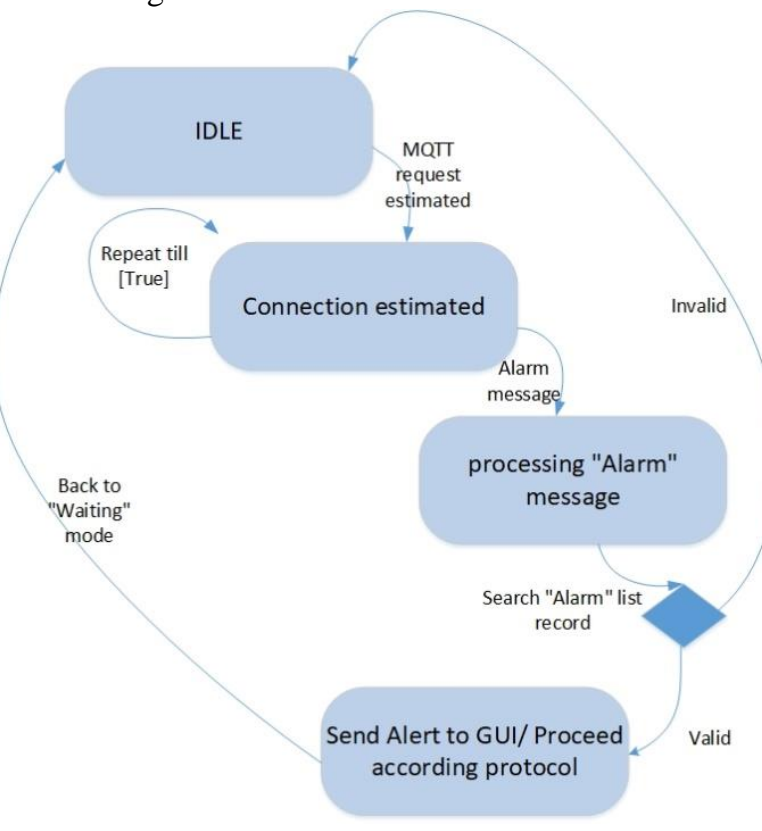

Figure 5. Smart home controller and "Outpost" solution intercommunication State Diagram

\section{CONCLUSIONS}

Technical application of represented conception is allowing to create periphery control and security network. Through using "Outpost" solution its is possible easy integrate current security concept to any smart house due flexibility of using of communication technology and messaging protocols. Main idea of using of existing solar powered street lights save the time and founds during

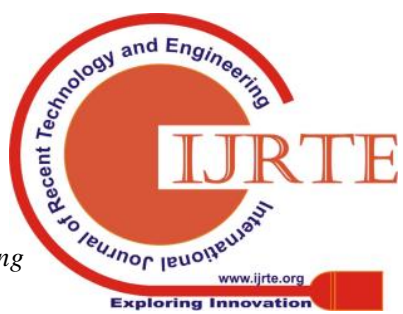


installation and maintaining. Organization of IoT security module itself on the Raspberry Pi 3B has no limits due it support Ethernet and Wi-Fi. Also experimentally selected 3 types of sensors is not a limit of the solution, adding different sensors is optional and variable. Remote and local controlling nowadays is have no limitation and supported by various types of hardware and software solutions. Network security between modules and smart homes depends on type of the communication technology.[10] Raspberry $\mathrm{Pi}$ as the controller solution showed great results during the experiments, which justify our expectations.

\section{ACKNOWLEDGEMENT}

This work was supported by the Technology development Program (S2648818) funded by the Ministry of SMEs and Startups (MSS, Korea)

\section{REFERENCES}

1. Rosslin John Robles, Tai-hoon Kim , A Review on Security in Smart Home Development, International Journal of Advanced Science and Technology Vol. 15, February, 2010

2. Md. Yousuf Hossain, Fabian Parsia George, Mita Halder, IOT based Automated Intrusion Detection System, nternational Journal of Computer Applications, Volume 180 - No.35, 2018, $0975-8887$

3. R. A. Messenger and J. Ventre, Photovoltaic systems engineering: CRC press, 2003.

4. R. W. Ritchie, Using Sunlight for Your Own Solar Electricity: Build Your Own System, Become Independent of the Grid, Domestic Photo Voltaics: Ritchie Unlimited Publications, 1999.

5. D. G. F. Sonnenenergie, Planning and installing photovoltaic systems: a guide for installers, architects and engineers: Earthscan, 2007.

6. Deepak.S.Kumbhar, Shubhangi M.Taur, H.C. Chaudhari, Shubhangi S.Bhatambrekar, IoT Based Home Security System Using Raspberry Pi-3, International Journal of Research and Analytical Reviews (IJRAR), Volume 6, Issue 1, 2019, ISSN 2349-5138

7. Hyoung-Ro Lee, Chi-Ho Lin, Design and Implementation of a Visitor Detection System using Hybrid Sensors, IEIE Transactions on Smart Processing and Computing, vol. 6, no. 5,2017

8. Aloÿs Augustin, Jiazi Yi, Thomas Clausen, William Mark Townsley, A Study of LoRa: Long Range \& Low Power,Networks for the Internet of Things, Sensors, MDPI, 2016

9. Taoufik Bouguera, Jean-François Diouris, Jean-Jacques Chaillout, Randa Jaouadi, Guillaume Andrieux, Energy Consumption Model for Sensor Nodes Based on LoRa and LoRaWAN, Sensors, MDPI, 2018

10. Cédric Lévy-Bencheton, Eleni Darra, Guillaume Tétu, Guillaume Dufay, Mouhannad Alattar, Security and Resilience of Smart Home Environments Good Practices and Recommendations, December 2015, ISBN: 978-92-9204-141-0 